\title{
LETTERS
}

\section{A pilot evaluation of the Zoned Asthma Action Plan (ZAAP) card}

\author{
James Findlay a, Jacqueline Parkes ${ }^{b}$, *Alison Ward`, Marylyn Richardson ${ }^{d}$ \\ ${ }^{a}$ General Practitioner, Rushden Medical Centre, Rushden, Northamptonshire, UK \\ ${ }^{b}$ Head of Nursing Developments, School of Health, The University of Northampton, UK \\ c Researcher, Centre for Health and Wellbeing Research, The University of Northampton, UK \\ d Senior Lecturer in Community Nursing, School of Health, The University of Northampton, UK
}

*Correspondence: Mrs Alison Ward, Centre for Health and Wellbeing Research, The University of Northampton, Park Campus, Boughton Green Road, Northampton, NN2 7AL, UK

Tel: +44 (0)1604 893559 E-mail: alison.ward@northampton.ac.uk

Received 28th November 2011; revised 5th December 2011;

accepted 5th December 2011; online 15th February 2012

(c) 2012 Primary Care Respiratory Society UK. All rights reserved

http://dx.doi.org/10.4104/pcrj.2012.00009

\section{Dear Sirs,}

Asthma exacerbations account for significant morbidity and contribute a disproportionate amount to the cost of asthma management. In the UK, f58.3million/year is spent on emergency care for asthma, and there were 79,794 emergency hospital admissions in 2008-09 of which an estimated $75 \%$ were avoidable through education and awareness of how to self-manage these events. ${ }^{1}$

Asthma self-management plans are fundamental to improvements in clinical outcomes for adult asthma, ${ }^{2-4}$ and are recommended as a means to promote self-management. However, a paradoxical situation exists in that, despite the UK Government's commitment to ensuring that everyone with a long-term condition has a personalised care plan by $2010,{ }^{5}$ many asthma patients do not receive an asthma action plan. ${ }^{6,7}$

In response to this, one of the authors (JF) developed a simplified and accessible self-management plan in the form of a credit card - the Zoned Asthma Action Plan (ZAAP) - which has been piloted and evaluated locally since 2002. The ZAAP card incorporates a traffic light zone response to self-care treatment options in line with current BTS/SIGN guidelines ${ }^{2}$ (see Figure 1). Thresholds for intervention are based on the patient's expected or best peak expiratory flow (PEF).

A Northamptonshire general practice with a specific interest in respiratory problems was identified as the pilot site for an audit evaluation. The ZAAP card and explanatory information were distributed to 162 participants identified by the practice's asthma register. Following a trial period, an evaluation questionnaire was completed by nurse practitioners and patients to assess and classify the patient's asthma symptoms within one of the four coloured zones (as identified within the ZAAP card).

Matched data were available in 156 cases. In 136 cases, the nurse practitioner and patient assessed the patient's asthma to be in the same zone; in seven cases the nurse assessed the patient's asthma to be less severe than the patient, and in 13 cases the nurse assessed the patient's asthma to be more severe than the patient. Where the nurse and patient's assessments differed, the nurse rated the asthma within the next zone, either one higher or lower.

Patients and nurse practitioners assessed the simplicity of understanding and ease of use of the ZAAP card in helping to manage asthma symptoms: $98 \% \quad(n=149)$ of patients and $96 \%$

Figure 1. Zoned Asthma Action Card

You are in the Green Zone if you can say YES to ALL of the following statements

- I seldom have any symptoms such as cough, wheeze, chest tightness or breathlessness

- I use my blue reliever no more than twice a week

- I am free of symptoms when resting or at night

- My peak flow is above (which is $85 \%$ of my expected reading)

This means that your asthma control is fine Remain on the same treatment until it is time for a routine review with your nurse or doctor You are in the Yellow Zone if you can say YES to ALL of the following statements

p I only get symptoms of asthma when hurrying

I I need my reliever in order to exercise normally

? I use my blue reliever no more than twice a day

I am free of symptoms when resting or at night

My peak flow is between and (between $75 \%$ and $85 \%$ of my expected reading) If you are often in this zone your maintenance therapy may need increasing - please follow your agreed plan or make a routine appointment
You are in the Orange Zone if ANY of the following statements apply to YOU

- My asthma is becoming more troublesome, - My blue reliever works less well than usual

- I am using my reliever three or four times a day

- I am getting mild symptoms at rest or at night

- My peak flow is between and (between $50 \%$ and $75 \%$ of my expected reading)

This means your asthma control has deteriorated Please see a nurse or doctor within 24 hours You may need a course of oral steroids

You are in the Red Zone if ANY of the following statements apply to YOU

- My asthma makes even walking difficult

- Sentences are hard to say in a single breath

- I am having to use my reliever every few hours

- I am getting severe symptoms at rest or at night

- My peak flow is less than (which is $50 \%$ of my expected reading)

This means you are having a severe asthma attack! This is a medical emergency - see your doctor immediately or go straight to Casualty 
$(n=149)$ of nurses reported that the ZAAP card was very or fairly easy to use; and $80 \% \quad(n=128)$ of patients and $78 \% \quad(n=123)$ of nurses believed it to be very or fairly helpful in managing patients' asthma levels.

Initial findings indicate that the ZAAP card is both easy to use and effective in supporting both the nurse and the patient's role within self-care management. Patients' own self-assessment of their level of asthma symptoms and the nurse practitioners' ratings were fairly similar, although the nurses tended to rate a greater number of patients as falling within the yellow, orange and red zones when compared with patients' own self-assessments.

This audit evaluation has found that patients' ability to selfassess their asthma symptoms within the correct coloured zone on the card is good. Furthermore, a higher proportion of patients assessed their asthma within the green zone when using the ZAAP card for a longer period of time, suggesting that those patients who have used the card for longer may understand and self-manage their asthma symptoms better. Whilst this suggests that the ZAAP card has a role to play in assisting patients to assess their symptoms accurately, there may be other factors which influenced the accuracy of self-reporting when using the card.

Further studies are needed to assess the efficacy and cost effectiveness of continued use of the ZAAP card. The fact that patients were not issued with a PEF meter led to PEF meter underuse. In future, by issuing all participants with a PEF meter, the relationship between accuracy of severity assessment with or without a PEF meter could be measured, and patient's acceptance of using a PEF meter to monitor and manage their symptoms explored further. Future research could also assess broader patient demographics and wider geographical areas as well as the ZAAP card's long term role in the management of asthma symptoms. This raises the importance of the need for patients to continually monitor and self-manage their asthma, and adds support to the need for self-management plans to improve clinical outcomes.

Conflicts of interest The authors declare that there are no conflicts of interest in relation to this article.

Contributorship JF was the creator of both the ZAAP card and audit questionnaire, and assisted with contextual and background information pertinent to the design of the card and the development of the project. AW was a researcher on the project and one of the authors of the article, writing the methods, findings and discussion section as well as providing editorial assistance on the final article. MR was a researcher on the project and one of the authors of the article, writing the introduction and literature review section as well as editorial assistance on the final article. JP was the project lead for the pilot evaluation and editor of the article.

Funding This project was funded by Nene Commissioning.

\section{References}

1. Asthma UK. The Asthma Divide. Inequalities in emergency care for people with asthma in England. London: Asthma UK; 2007. 18 p.

2. The British Thoracic Society \& Scottish Inter-collegiate Guideline Network British guideline on the management of asthma: quick reference guide. London: The British Thoracic Society; 2011 May. 24 p.

3. Global Initiative for Asthma. Pocket guide for asthma management and prevention: a pocket guide for physicians and nurses. Global Initiative for Asthma; 2003. 28 p.

4. Asthma UK Scotland. The Asthma Charter. Edinburgh: Asthma UK Scotland 2004. 2 p.

5. Darzi A. High Quality Care for All: NHS Next Stage Review Final Report Norwich: Department of Health; 2008 June. 92 p

6. Price D, Wolfe S. Delivery of asthma care: patients' use of and views on healthcare services as determined from a nation-wide interview survey. Asthma J 2000;5:141-4.

7. Haughney J, Barnes G, Partridge M, Cleland J. The Living and Breathing Study: a study of patients views of asthma and its treatment. Prim Care Respir J 2004;13:28-35. http://dx.doi.org/10.1016/j.pcrj.2003.11.007

Available online at http://w w w.thepcrj.org 